\title{
Percepción de inseguridad y su influencia en el bienestar subjetivo de los habitantes de la Zona Conurbada del Valle de México
}

\section{Infiencie of perception of insegurity on the subjective well-being of the inhabitants of the Conurbated Zone of the Valley of Mexico}

\author{
DOI: $10.46932 / \mathrm{sfjdv2n5-046}$
}

Received in: Oct 1st, 2021

Accepted in: Dec 30th, 2021

\author{
Fabiola Olvera Aldana \\ Doctora en Ciencias Políticas y Sociales. \\ Álvaro Obregón, C.P. 01030, CDMX. \\ E-mail: F.olvera.aldana@gmail.com
}

\section{RESUMEN}

En México, el Estado ha fallado al mantener el orden público, promover el bien común, disminuir la delictividad y la percepción de inseguridad en su población, como lo muestran las estadísticas nacionales donde el $67.2 \%$ de la población señala que el principal problema del país es la seguridad. Esto ha permitido que el llamado Segundo Estado, tome control y promueva sus acciones delictivas, provocando que la población se mantenga en un estado contante de alerta y de modificación de sus hábitos, buscando mantenerse a salvo de la delincuencia. Este fenómeno es aún frecuente en las grandes ciudades, sobre todo en los espacios donde existen grandes grupos poblacionales, como lo es el borde entre la Ciudad de México y el Estado de México. El presente análisis documental pretende exponer la forma en que la delictividad ha cambiado en esta zona, describiendo como han cambiado los índices de delincuencia y a su vez, como esto ha influenciado en la percepción de seguridad y el bienestar de las personas que viven o se mueven por este espacio, sobre todo, en las adaptaciones que han tenido que realizar para no ser víctimas de la violencia y delincuencia que se desarrolla en espacios públicos y el transporte.

Palabras Clave: Inseguridad, Bienestar, Delito, Valle de México, Victimización.

\begin{abstract}
In Mexico, the State has failed to maintain public order, promote the common good, reduce crime and the perception of insecurity; national statistics shown that $67.2 \%$ of the population said that the country's main problem is the security. This has allowed the so-called Second State to take control and promote criminal activities, causing the population to remain constantly alert and changing their habits, seeking to keep themselves safe from crime. This phenomenon is still common in large cities, especially in areas where there are large population groups, such as the border between Mexico City and the State of Mexico. This documental nalysis aims to show how crime has changed in this area, describing the crime rates's change and how that have a influence in the perception of security and well-being of people who live or move through this area, especially the adaptations they have had to make in order not to be victims of violence and crime in public spaces. and transportation.
\end{abstract}

Keywords: Insecurity, Welfare, Crime, Valley of Mexico, Victimization. 


\section{INTRODUCCIÓN}

México es un país cargado de contrastes, en donde el Estado ha fallado al intentar mantener la ley, el orden público, la promoción del bien común, la gestión y legislación sobre los asuntos que afectan a la población, la emisión de moneda, la regulación del mercado, la distribución de la riqueza, entre otras. Por lo tanto, no es de extrañar que en un país en el que cada 15 minutos hay 6 personas asesinadas (Lozano y Sánchez, 2008), el Estado se encuentre experimentado un proceso de retraimiento, derivado del surgimiento de nuevas instituciones paralelas que, de igual forma, organizan la existencia de los habitantes.

Una de dichas instituciones paralelas, que se han instalado en todas las esferas de la vida social, económica y policía del país es el denominado "Segundo Estado", que se conforma como una estructura de control estatal y que se caracteriza por el uso del miedo, la violencia y la realización de actos criminales en extremo transgresores para controlar a la sociedad. Está compuesto de sus propias instituciones, leyes, relaciones e instrumentos, que a través de la violencia y el control que ejerce sobre las personas reemplaza al sistema estatal que, si bien está legitimado, se encuentra débil y enfermo (Segato, 2016a). De igual forma, tiene sus propias normas que protegen a quienes les siguen y castigan a los que no.

\section{ANÁLISIS}

La máxima expresión conductual del control y el poder del Segundo Estado, es la violencia ejercida en los espacio público y abierto, muestra la intención de determinados grupos por exhibir su capacidad de dominio sobre la población. Ello, aunado al hecho de que la criminalidad pareciera actuar en red y contar con una articulación precisa, muestra la existencia de una estructura organizada que controla y da forma a la vida social de manera subrepticia y al margen de la ley. Utiliza la estrategia clásica del poder soberano para reproducirse, consistente en divulgar que puede actuar más allá de la norma (Segato, 2016a).

Particularmente, el crimen organizado es una de las instituciones que, en América Latina, se ha constituido como un Segundo Estado, teniendo atribuciones paralelas al Estado tradicional. Se ha constituido como el poder soberano, que impacta sobre las esferas sociales y en la individualidad física, moral y psicológica de las personas que viven en un territorio (Segato, 2016b). Por lo tanto, no es de extrañar que en espacios donde se presenten carencias, desigualdad y que haya sido abandonado por el Estado tradicional, el Segundo Estado tenga el poder y ejerza violencia sobre los habitantes.

El Según Estado ha realizado estragos en México, que se reflejan en las cifras de incidencia delictiva; por ejemplo, el Área Metropolitana del Valle de México se ha visto más afectada en los últimos dos años, presentando una tasa de prevalencia delictiva de 35.9 por cada 100000 habitantes; aun así, la 
cifra negra del delito es de un $93.2 \%$ de los delitos cometidos, pero que no fueron denunciados. Esto afecta directamente en la economía individual, pues al ser víctima del delito representa una pérdida económica del $61 \%$ del ingreso, pues se realizan cambios de infraestructura al colocar cerradura, candados, puertas o ventanas, poner rejas o bardas, entre otras; a cada hogar de México la inseguridad y el delito representan un gasto de 286.3 mil millones de pesos es decir el 1.54\% de PIB (INEGI, 2019).

Lo anterior, permite entender las cifras asociadas a la percepción sobre la seguridad pública, pues a nivel nacional el $67.2 \%$ de la población de 18 y más piensan que la inseguridad es el problema más importante del país. No es de extrañar que la percepción de inseguridad haya aumentado alrededor de 10 puntos del 2013 a 2019, sin importar si se hace referencia a la colonia, municipio o estado, pues en los tres espacios se presentan un incremento similar (Sistema del transporte colectivo, 2020).

De manera general, en México el Segundo Estado ha encontrado espacios específicos para imponerse. En particular, el Estado de México y el borde con la Ciudad de México se ha convertido en una zona que ha incrementado los índices delictivos y donde las personas han tenido que modificar su día a día para sobrevivir, afectando la interacción social, crecimiento económico y el bienestar.

\subsection{FRANJA DEL VALLE DE MÉXICO: ESPACIO DE VIOLENCIA Y PERCEPCIÓN DE INSEGURIDAD}

Las cercanías al metro Río de los Remedios (Sistema del transporte colectivo, 2020) es un cúmulo de 25 colonias, en las que se permean los contrastes y altos niveles de desigualdad existentes; geográficamente, es la barrera entre la Ciudad de México y el Estado de México, espacio en el que comparten territorio las siempre turbulentas Ecatepec de Morelos, Nezahualcóyotl y Gustavo A. Madero (CONAGUA, 2019). Por lo que no es de extrañar que las personas que habitan en las cercanías al metro hayan desarrollado estrategias y tácticas de supervivencia después de dos décadas en condiciones de hacinamiento, sin servicios básicos o pavimento, banquetas e iluminación y con un Río que se ha convertido en aguas negras y residuales, provocando que la transformación del estilo de vida, como resultado de la expansión de la ciudad o mancha urbana, generando la aparición de las formas de vida adaptadas al contexto (Aguilar, 2021).

En cuanto al número de personas que habitan en dicho espacio, en la tabla 1 se desglosan las estadísticas poblacionales. 
Tabla 1. Datos sociodemográficos generales de la Franja del Valle de México

\begin{tabular}{|c|c|c|c|c|c|c|c|}
\hline \multirow{2}{*}{$\begin{array}{l}\text { Alcaldía } \\
\text { municipio }\end{array}$} & \multicolumn{3}{|c|}{ Población } & \multicolumn{3}{|l|}{ Edad } & \multirow{2}{*}{$\begin{array}{l}\text { Viviendas } \\
\text { Total }\end{array}$} \\
\hline & Hombres & Mujeres & Total & $\begin{array}{l}\text { Rango (0- } \\
14)\end{array}$ & $\begin{array}{l}\text { Rango (15- } \\
65)\end{array}$ & $\begin{array}{l}\text { Rango }(65 \\
\text { y más) }\end{array}$ & \\
\hline $\begin{array}{l}\text { Gustavo } \quad \text { A } \\
\text { Madero }\end{array}$ & 17946 & 19322 & 37294 & 8876 & 24486 & 3217 & 10675 \\
\hline $\begin{array}{l}\text { Ecatepec de } \\
\text { Morelos }\end{array}$ & 50855 & 53307 & 104269 & 25737 & 71525 & 5240 & 29501 \\
\hline Nezahualcóyotl & 22487 & 24686 & 47217 & 8302 & 33976 & 3886 & 14771 \\
\hline Totales & 91288 & 97315 & 188780 & 42915 & 129987 & 12343 & 54947 \\
\hline
\end{tabular}

Fuente: Elaboración propia con información del Instituto de Estadística y Geografía [INEGI] (2010).

Es un punto medio afectado por un cúmulo de problemas sociales multifactoriales que cuenta con una sobrepoblación y migración latente que ha ido agravándose por el abandono del Estado y políticas públicas, desarrollando entre sus habitantes un comportamiento de frontera por un segundo actor social que ha tratado de tomar el control mediante la percepción de inseguridad y amenazas, formando un disciplinamiento y posterior naturalización de convivencia social con células delictivas del crimen organizado (ONUDD, 2020).

Lo anterior, ameritó que la zona sea una de las primeras en contar con la presencia de la Guardia Nacional con el operativo "Cordones Seguros” (Campos, 2019); específicamente en las colonias Valle de Aragón, en Nezahualcóyotl, El Chamizal y Granjas Valle de Guadalupe, en Ecatepec de Morelos, y San Felipe de Jesús, en Gustavo A. Madero (Milenio Digital, 2019), estando todas ellas en las inmediaciones del metro Río de los Remedios.

Sin embargo, siguen presentándose elevadas tazas de delito en dicha zona. Podemos ver como en la alcaldía Gustavo A. Madero (Secretariado Ejecutivo del Sistema Nacional de Seguridad Pública, 2019), entre 2016 y 2017 se dio un aumento significativo en el número de robos y de 2017 a 2018 un aumento en el narcomenudeo y las violaciones, además de destacarse por el elevado número de casos de violencia familiar.

Para Ecatepec de Morelos, los datos que destacan son el alto número de homicidios, que tuvieron un alza de 2016 a 201; por su parte, los robos aumentaron casi la mitad entre 2015 y 2018. Por su parte, en Nezahualcóyotl, se distinguen que el número de lesiones casi se triplicaron, los robos aumentaron casi un $25 \%$ y las violaciones se incrementaron en un 50\% entre 2015 y 2018 (tabla 2). 
Tabla 2. Condensado de número de delitos en la zona y porcentaje correspondiente a cada alcaldía

\begin{tabular}{|c|c|c|c|c|c|c|c|c|c|}
\hline & & Robo & $\begin{array}{l}\text { Violenci } \\
\text { a } \\
\text { familiar }\end{array}$ & $\begin{array}{l}\text { Lesione } \\
\text { S }\end{array}$ & $\begin{array}{l}\text { Homicidio } \\
\text { S }\end{array}$ & $\begin{array}{l}\text { Narcomenude } \\
\text { o }\end{array}$ & $\begin{array}{l}\text { Violació } \\
\text { n }\end{array}$ & $\begin{array}{l}\text { Secuestr } \\
\text { o }\end{array}$ & $\begin{array}{l}\text { Feminicidi } \\
\text { o }\end{array}$ \\
\hline \multirow{4}{*}{$\begin{array}{l}201 \\
5\end{array}$} & TOTAL & 26811 & 6641 & 2577 & 886 & 692 & 358 & 42 & 13 \\
\hline & GAM & $33 \%$ & $29 \%$ & $58 \%$ & $28 \%$ & $33 \%$ & $22 \%$ & $19 \%$ & $23 \%$ \\
\hline & $\begin{array}{l}\text { Ecatepe } \\
\text { c }\end{array}$ & $50 \%$ & $57 \%$ & $22 \%$ & $46 \%$ & $56 \%$ & $68 \%$ & $69 \%$ & $54 \%$ \\
\hline & Neza & $17 \%$ & $14 \%$ & $20 \%$ & $26 \%$ & $11 \%$ & $10 \%$ & $12 \%$ & $23 \%$ \\
\hline \multirow{4}{*}{$\begin{array}{l}201 \\
6\end{array}$} & TOTAL & 29241 & 7772 & 2004 & 938 & 598 & 675 & 44 & 18 \\
\hline & GAM & $28 \%$ & $25 \%$ & $71 \%$ & $25 \%$ & $30 \%$ & $6 \%$ & $16 \%$ & $33 \%$ \\
\hline & $\begin{array}{l}\text { Ecatepe } \\
\text { c }\end{array}$ & $55 \%$ & $55 \%$ & $13 \%$ & $52 \%$ & $56 \%$ & $92 \%$ & $48 \%$ & $56 \%$ \\
\hline & Neza & $16 \%$ & $21 \%$ & $16 \%$ & $22 \%$ & $15 \%$ & $2 \%$ & $36 \%$ & $11 \%$ \\
\hline \multirow{4}{*}{$\begin{array}{l}201 \\
7\end{array}$} & TOTAL & 37697 & 7595 & 2077 & 1377 & 824 & 46 & 38 & 11 \\
\hline & GAM & $28 \%$ & $27 \%$ & $72 \%$ & $20 \%$ & $49 \%$ & $72 \%$ & $11 \%$ & $36 \%$ \\
\hline & $\begin{array}{l}\text { Ecatepe } \\
\text { c }\end{array}$ & $60 \%$ & $52 \%$ & $12 \%$ & $64 \%$ & $41 \%$ & $0 \%$ & $47 \%$ & $45 \%$ \\
\hline & Neza & $13 \%$ & $21 \%$ & $15 \%$ & $15 \%$ & $11 \%$ & $28 \%$ & $42 \%$ & $18 \%$ \\
\hline \multirow{4}{*}{$\begin{array}{l}201 \\
8\end{array}$} & TOTAL & 35987 & 7936 & 1702 & 1220 & 1340 & 649 & 45 & 34 \\
\hline & GAM & $34 \%$ & $27 \%$ & $80 \%$ & $21 \%$ & $63 \%$ & $21 \%$ & $38 \%$ & $18 \%$ \\
\hline & $\begin{array}{l}\text { Ecatepe } \\
\text { c }\end{array}$ & $50 \%$ & $41 \%$ & $12 \%$ & $61 \%$ & $28 \%$ & $22 \%$ & $40 \%$ & $56 \%$ \\
\hline & Neza & $16 \%$ & $33 \%$ & $8 \%$ & $18 \%$ & $9 \%$ & $56 \%$ & $22 \%$ & $26 \%$ \\
\hline \multirow{4}{*}{$\begin{array}{l}201 \\
9\end{array}$} & TOTAL & 23993 & 6240 & 1053 & 817 & 676 & 546 & 58 & 11 \\
\hline & GAM & $27 \%$ & $27 \%$ & $74 \%$ & $20 \%$ & $55 \%$ & $13 \%$ & $26 \%$ & $45 \%$ \\
\hline & Eca & $53 \%$ & $47 \%$ & $15 \%$ & $60 \%$ & $36 \%$ & $46 \%$ & $14 \%$ & $36 \%$ \\
\hline & $\mathrm{Neza}$ & $19 \%$ & $26 \%$ & $10 \%$ & $20 \%$ & $9 \%$ & $41 \%$ & $60 \%$ & $18 \%$ \\
\hline
\end{tabular}

Fuente: Secretariado Ejecutivo del Sistema Nacional de Seguridad Pública (2019).

Las elevadas cifras, sobre todo en Ecatepec, ha provocado que las personas que viven en las colonias cercanas o las que tiene que movilizarse usando el transporte de dicho espacio, se vean obligadas a implementar estrategias que les permitan evitar la victimización.

El espacio público y las zonas de gran movimiento población, por su cualidad de frontera entre la capital del país y el Estado de México (INEGI, 2017), han perdido la tranquilidad y la percepción de neutralidad, producto generado por el Segundo Estado, pues se ha encargado de dejar en el colectivo el ejercicio de la violencia y que las personas sepan que siempre pueden ser víctimas (Zúñiga, 2014).

Sin duda, México es un país de contraste, pues si bien presenta altos índices de delitos y una elevada percepción de inseguridad, se mantiene presente una calificación de 8.3 de 10 en cuanto al bienestar, encontrándose que el $97.8 \%$ de la población se encuentra satisfecha con su vida. Cabe señalar, que esta medición bienestar se refiere a la perspectiva sobre su calidad de vida, poder adquisitivo, relaciones personales, actividades, vivienda, estado de salud, logros en la vida, perspectiva a futuro, vecindario, tiempo libre, percepción sobre el país y finalmente la seguridad ciudadana (Gobierno de México, 2019). Sin embargo, a pesar de que existen estos elevados niveles de bienestar subjetivo, también se destaca que la satisfacción con la seguridad ciudadana disminuyó de 5.4 a 5.3 del 2018 al 2019 (INEGI, 2019). Es decir, los mexicanos están satisfechos con sus vidas, pero se siente cada vez más inseguros. 


\section{CONCLUSIONES}

La inseguridad implica retos para las personas que habitan en zonas como la franja del valle de México, pues ha ocasionado que se vean forzados a realizar cambios en ciertas actividades y generar estrategias que les permitan sobrevivir a la adversidad de la realidad violenta en la que viven todos los días (Blari, 2005). Estas estrategias están "alejadas" de la normalidad y en su mayoría son de carácter individual (Arteaga, 2003). Intentan hacer frente a la violencia e inseguridad, con pocas expectativas sobre la labor de protección de las autoridades policiales, pues se encuentran desprotegidas y no se confía en ellos ${ }^{5}$.

En esta zona y a nivel nacional, los cambios conductuales que las personas tuvieron que realizar como resultado de la percepción de inseguridad, principalmente fueron: permitir que sus hijos menores salieran de casa (71\%), dejaron de usar joyas al salir a la calle (60.9\%), evitar salir de noche (53.4\%) y llevar dinero en efectivo $(50.4 \%)$.

Otras de las modificaciones a la rutina que se vieron afectadas por la inseguridad, incluyen el evitar salir a caminar, no tomar un taxi, no salir a correr, evitar visitar a parientes o amigos, no asistir a espacios públicos e incluso, modificar la rutina para hacer uso del transporte público. Una de las consecuencias principales y más peligrosas de este fenómeno, es que las personas normalicen y adopten los comportamientos violentos como medio de sobrevivencia, usando las actividades ilícitas un medio de ascenso social, provocando que un círculo vicioso que incremente la delincuencia (Castillo y Castro, 2011).

Lo anterior es relevante porque la ciudad constituida a partir de los espacios públicos, privados, políticos y artísticos expresa las formas de vida, que a su vez reflejan la cultura (Aguilar, 2021), por lo que debemos cuestionarnos, el tipo de naturalización cultural que se está generando a partir de la inseguridad en México. 


\section{REFERENCIAS}

Aguilar, I. (2021). La ciudad contemporánea: Identidad y patrimonio. South Florida Journal of Development, 2 (4), 6160-6168.

Arteaga, N. (2003). El espacio de la violencia: un modelo de interpretación social. Sociológica,18 (52), $119-145$

Blari, E. (2005). Memorias de violencia, espacio, tiempo y narración. Controversia, 185, 9-19.

Castillo, V. y Castro, C. (2011). El rostro de la violencia social y estructural: la delincuencia y la pobreza como expresiones distintas de una vulnerabilidad común. Rev. Ciencias Sociales. 133-134, 113-124.

Campos, G. (2019). De madrugada, Guardia Nacional realiza operativos en Ecatepec. México: El Sol de México; Recuperado de: https://www.elsoldemexico.com.mx/metropoli/valle-de-mexico/de-madrugadaguardia-nacional-realiza-operativos-en-ecatepec-4144220.html

CONAGUA. (2019). El Río de los Remedios: Una visión ambiental. Recuperado de: https://agua.org.mx/wp-content/uploads/filespdf/doc_pdf_6738.pdf

Gobierno de México. (2019). Se mantiene en 8.3 de 10 la calificación sobre el bienestar de la población adulta urbana en nuestro país en julio. México: Gobierno de México. Recuperado de: https://www.gob.mx/shcp\%7Cgacetaeconomica/articulos/se-mantiene-en-8-3-puntos-el-nivel-debienestar-subjetivo-de-la-poblacion-adulta-urbana-en-nuestro-pais-en-julio

INEGI. (2017). Encuesta origen-destino en Hogares de la Zona Metropolitana del Valle de México. México: INEGI, UNAM, Gobierno de CDMX y Gobierno de EDOMEZ.

INEGI. (2010). Censo de población y vivienda. Recuperado de: https://www.inegi.org.mx/programas/ccpv/2010/

INEGI. (2019). Encuesta Nacional de Victimización y Percepción sobre Seguridad Pública. México: INEGI.

INEGI. (2019). Indicadores de bienestar autoreportado de la población urbana, cidras del mes de julio de 2019. México: INEGI.

Lozano, M. y Sánchez, D. (2008). Filosofía y ciudadanía. España: McGraw Hill.

Milenio Digital. (2019). Ecatepec dona predio para base de Guardia Nacional [Internet]. México: Milenio. Recuperado de: https://www.milenio.com/ politica/gobierno/ecatepec-dona-predio-base-guardia-nacional

Oficina de las Naciones Unidas contra la Droga y el Delito (ONUDD). (2020). Crimen Organizado Trasnacional. Recuperado de: https://www.unodc.org/ropan/es/organized-crime.html

Secretariado Ejecutivo del Sistema Nacional de Seguridad Pública. (2019). Incidencia delictiva del Fuero Común, nueva metodología. Recuperado de: https://www.gob.mx/sesnsp/acciones-yprogramas/incidencia-delictiva-del-fuero-comun-nueva-metodologia?state=published 
Segato, R. (2016a). La escritura en el cuerpo de las mujeres asesinadas en Ciudad Juárez Territorio, soberanía y crímenes de segundo Estado. Buenos Aires: Tinta Limón.

Segato, R. (2016b). La guerra contra las mujeres. Madrid: Traficantes de sueños.

Sistema de Transporte Colectivo. (2020). Línea B. Metro CDMX. Recuperado de: https://www.metro.cdmx.gob.mx/la-red/linea-b

Zúñiga, M. (2014). Las mujeres en los espacios públicos: entre la violencia y la búsqueda de libertad. Región y sociedad. 4, 77-100. 